\title{
Influence of a mutltistrand cable design on its quench development process and stability*
}

\author{
Vitaly S. Vysotskyt5, Alexei V. Korobkot, Yuri A. llyint, Gert B.M. \\ Mulder $\uparrow$ and Herman H.J. ten Kate‡
}

\author{
†Institute of Superconductivity and Solid State Physics of Russian Research Center \\ 'Kurchatov Institute', 123182, Kurchatov Square 1, Moscow, Russia \\ ¥University of Twente, Applied Superconductivity Center, PO Box 217, 7500 AE \\ Enschede, The Netherlands
}

\section{Received 2 December 1996; revised 21 April 1997}

The quench development process was studied with two six-strand superconducting cable samples with insulated strands and different cabling design. One sample was the traditional 'six-around-one' cable, the other was of the 'round-braid' type. Quench was initiated by a heating pulse applied to a single strand. A significant difference was observed in the current redistribution among strands due to the different cabling designs. It has an important influence on the stability of multistrand cables. A new concept of the 'single-strand stability' was proposed and corresponding stability criteria were offered. A possible way to improve cable stability by using special cabling design is discussed. (C) 1997 Elsevier Science Ltd.

Keywords: superconducting cables; quench; stability

\begin{abstract}
Multistrand superconducting cables are of wide use in many applications of superconductivity. Along with many advantages such as low AC losses, easy production and coil forming processes, etc., some common problems are associated with these cables. Most important is the reduction of stability of such cables, mainly at non-steady state conditions ${ }^{1-3}$. Quench current of the cable appears to be significantly less than the sum of critical currents of each of the strands. Several reasons for such behaviour have been considered previously ${ }^{1-3}$.

In many cases the quench of the multistrand cable may start from a single-strand quench ${ }^{4}$, but the single-strand quench may or may not lead to the quench of the entire cable $e^{4,5}$. Particularly, the process of the current redistribution from the quenched strand to adjacent strands has an influence on the subsequent quench development. This current redistribution depends on inductive coupling between adjacent strands and may be changed by changing the cable design.
\end{abstract}

\footnotetext{
*The part of this work detailing the test of 'round-braid' cable was the subject of the Master's Thesis of A.V. Korobko. All experiments were performed at the Applied Superconductivity Center of Twente University

§Present address: Faculty of Engineering, Kyushu University, 36, 6-10-1 Hakozaki, Higashi-ku, Fukuoka 812-81, Japan TPresent address: Philips Medical Systems, PO Box 10.000 5680 DA Best, The Netherlands
}

In this paper we compared quench development processes in two six-strand cables with different cabling designs. The goal of the study was to determine the influence of the design of cables on the quench development process and their stability. A new concept of the 'singlestrand stability' was suggested and discussed.

\section{Samples and experimental results}

Both sample cables have insulated strands with $\mathrm{CuNi}$ matrix. One of the samples (1) has traditional 'six-aroundone' twisting with a central non-superconducting strand; the second sample (2) is a 'round-braid' cable. The difference in the cable design is illustrated in Figure 1. During sixaround-one cabling all six strands are twisted in the same direction around the central non-superconducting strand. The distance between each strand is the same along the entire cable.

During round-braid preparation, odd and even strands are twisted around the central strand in opposite directions with interlacing. The distance between strands in the same triplet remains constant, but changes between strands in different triplets. During a twist pitch each strand from one triplet is in direct contact with all strands from another triplet. In some cases the central strand may not be_used. This roundbraid design may be visualized by taking the example of RF shields on cables used for TV aerials. This is a standard cabling technique. 


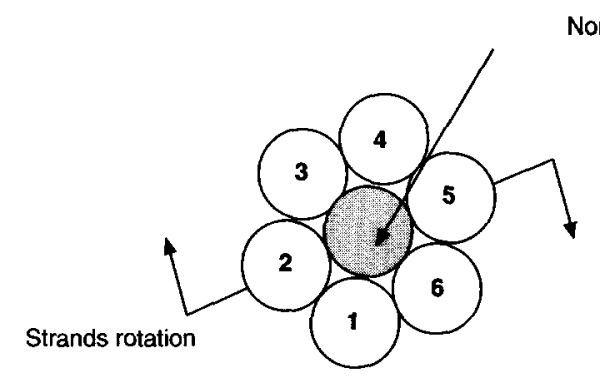

"Six-around-one" cabilng

Non-superconducting strand

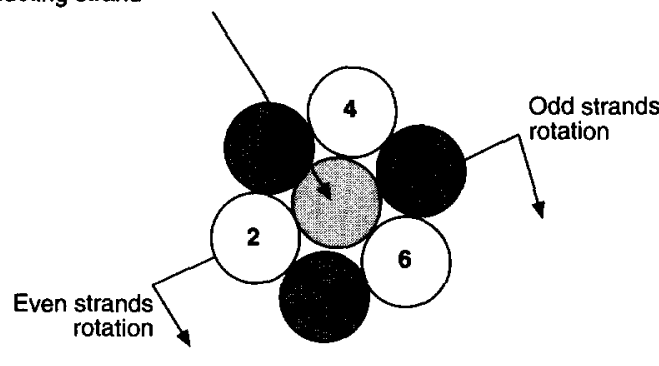

"Round braid" cabling

Figure 1 Cable design of the samples

Parameters of the strands used in the sample cables are listed in Table 1.

The measuring technique and the test results of sample 1 were described in details elsewhere ${ }^{5}$. Sample 2 was subjected to similar testing. Test coils were wound from both samples. At one end, the sample was untwisted and each strand was provided with pick-up coils to measure current in it. During measurements, DC current was supplied to the coil, the quench was initiated using a heater on one strand and signals from pick-up coils were recorded by a digital oscilloscope. Integrated signals from pick-up coils gave us the current in each strand.

It was found that depending on the initial current there are three types of quench development processes ${ }^{5}$ which are similar for both cables. Currents in strands versus time are shown for both samples in Figures 2 and 3 for different initial total currents. In all cases, the quench was initiated by the heater on strand 1 .

If the total current is below some certain level only one strand is quenched and its current is redistributed to another strands without their being quenched (Figures $2 a$ and $3 a$ ). The total cable current remains unchanged. This was referred to previously ${ }^{5}$ as a 'redistribution'. When the total current exceeded some certain level $\left(\sim 660 \mathrm{~A}\right.$ or $\sim 0.22 I_{\mathrm{c}}$ for sample 1 and $\sim 240 \mathrm{~A}$ or $\sim 0.29 I_{\mathrm{c}}$ for sample 2 ) the increase of current in adjacent strands leads to their quench at some current level and to the increase of current in other strands. Eventually, it leads to total cable quench (Figures $2 b$ and $3 b$ ); it is the so-called 'slow quench' region'. Further initial current increase leads to a very fast quench development process known as the 'fast quench' phenomenon. These quench development processes were investigated $^{5-7}$ and explained $\mathrm{d}^{5,8}$ previously.

In spite of the general similarity of the quench development processes in both cables, there is one important difference: once the first strand is quenched its current is redistributed in a different number of strands. In sample 1 (sixaround-one) the current mostly redistributed to two strands (2 and 6), while in the sample 2 (round-braid) current mostly redistributed to three strands (2, 4 and 6 ). We note that strand 4 is furthest from the quenched strand 1 . During quench of sample 1 practically no current is redistributed to this strand, while during quench of sample 2 this strand accepts about one-third of the current dropped from strand 1.

\section{Discussion: the single-strand stability of multistrand superconducting cables and its dependence on cabling}

The fact that in the six-around-one cable with insulated strands only two adjacent strands show a significant increase of current has been explained previously ${ }^{5}$, and the first attention to similar phenomena was paid by Rusinov

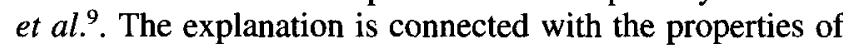
the inverse matrix of mutual inductances which is diagonally dominant and therefore only the nearest inductances have a strong reaction to a change of current in the first one. Inductances placed far from the first are shielded. A small difference between the inductive coupling coefficient leads to a large difference in current redistribution; it is just mutual inductance that is responsible for current redistribution from the quenched strand to the others.

The mutual inductance between two adjacent strands is inversely proportional to the distance between them ${ }^{7}$. In the six-around-one cable, distances between all strands are constant and two adjacent strands have the highest mutual inductances. In the round-braid cable, even strands come close to a certain odd strand during twist pitch, while distances between all even strands (and between all odd strands) remain unchanged. This is why inductive coupling is the same between certain strands in one triplet and all other strands in the other triplet. The inductive coupling between strands in the same triplet is less than between strands from different triplets in the round-braid cable.

This explains the difference in current redistribution in our samples. Current from the quenched strand always comes to those strands with which it has highest mutual inductance. In the six-around-one cable, strand 1 has the highest mutual inductance with strands 2 and 6 and the dropped current is divided between the two strands. In the round-braid cable, strand 1 has highest mutual inductance with strands 2, 6 and 4 and the current dropped from strand

Table 1 Parameters of strands used in the sample cables

\begin{tabular}{|lllll}
\hline Sample number & Type & $\begin{array}{l}\text { Strand diameter, } \\
\mathrm{mm}\end{array}$ & Filament number & $\begin{array}{l}\text { Matrix resistivity, } \\
\mu \Omega \mathrm{m}\end{array}$ \\
\hline 1 & $\begin{array}{l}\text { Six-around-one } \\
\text { cable } \\
\text { Round-braid cable }\end{array}$ & 0.5 & 200 & 13.5 \\
2 & 0.2 & 574 & 25 \\
\hline
\end{tabular}



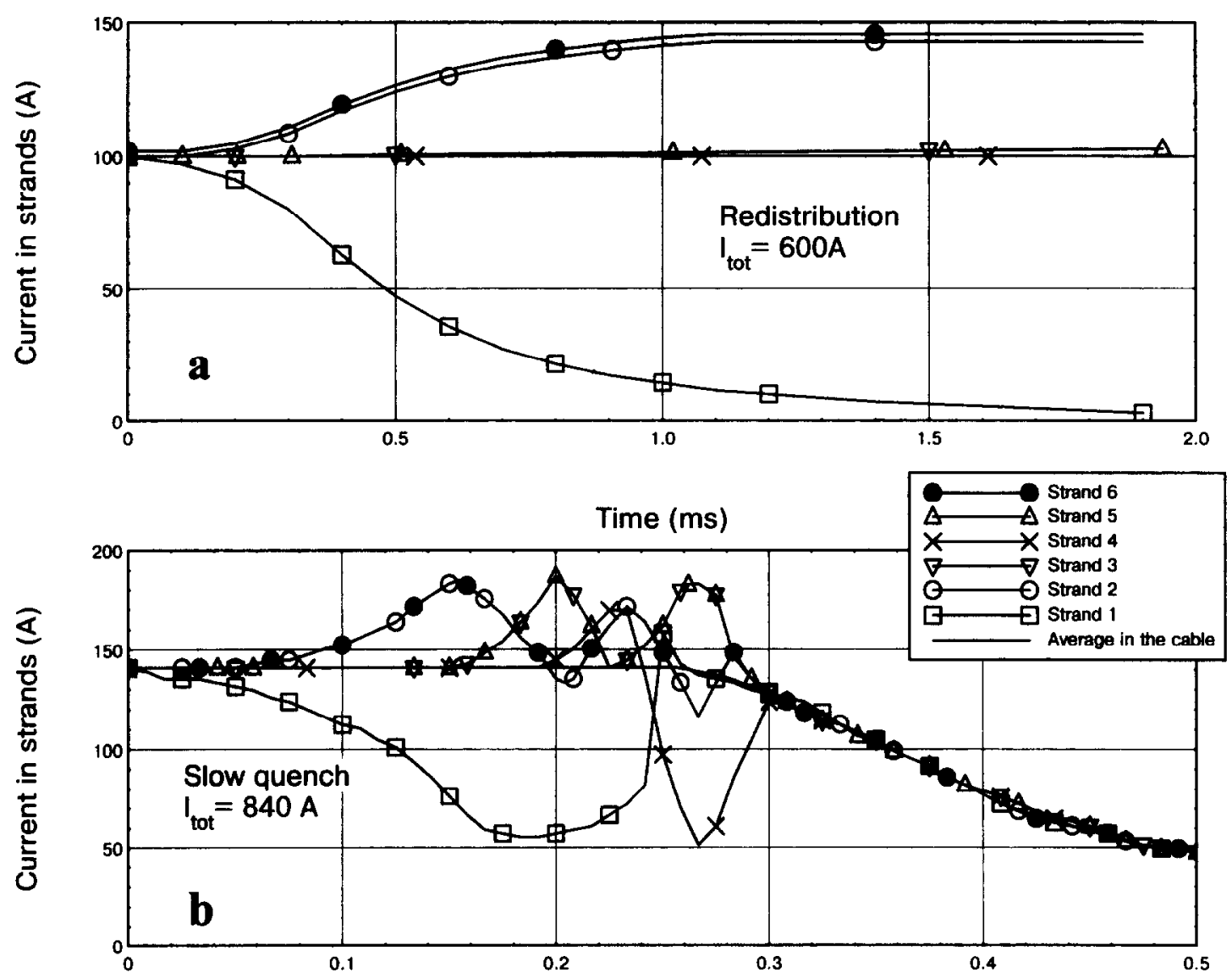

Time (ms)

Figure 2 Quench development process in sample \#1 (six-around-one cabling): (a) redistribution process; (b) slow quench process

1 is divided between three strands. This is illustrated in Figure 4, where the redistribution process is shown for current normalized to the initial value. One can see that in the six-around-one cable, the current rise is about $50 \%$, while in the round-braid cable current rise is only $33 \%$.

On the other hand, the conditions at which total cable quench starts are different in our samples. This is illustrated in Figure 5, where slow quench development processes in both cables are shown for the currents in the strands normalized to their critical current values. The quench starts from the same relative current. One can see that before the quench of the next strands occurs (that leads to entire cable quench), current in the round-braid cable may rise by $12 \%$ of its initial value while current in the six-around-one cable rises by only $8 \%$ of $I_{c}$, or 1.5 times less. This agrees exactly with the relationship between the number of strands in which the current redistributes if one single-strand quenches.

This difference in the current redistribution leads to very important consequence for the consideration of cable stability, meaning the stability of the cable in relation to the possible quench of one single strand. Let us call it 'singlestrand stability' or SS stability. The quench of one single strand of a multistrand superconducting cable may occur due to many reasons, e.g. a weak point in the strand, the current in a certain strand exceeding the average level ${ }^{4}$ in the cable, etc. The cable should be stable to this event, i.e. quench of one strand should not lead to the quench of other strands and to entire cable quench. In particular, this is important for cables with insulated strands or with strands with highly resistive matrix and special covering, like chromium covering of strands in $\mathrm{Nb}_{3} \mathrm{Sn}$ based CICC. In such cables direct current sharing from the quenched strand to other strands is difficult and the current redistribution to other strands has an electrodynamic origin ${ }^{10}$. Thus, SS stability may be also referred to as 'electrodynamic stability'.

Let us consider the stability criteria and the limiting current for SS stability of a multistrand superconducting cable with insulated strands. Let the cable have an average current per strand $I_{\text {str }}$ and a critical current of the strands $I_{\mathrm{c}}$. For consideration of the single-strand stability, the most important parameter is the number of strands to which the current from the quenched strand will redistribute; this is denoted as $m$. This parameter does not depend on $N$, the number of strands in the cable, for multiple twisting cables and Rutherford type cables. For round-braid cables this parameter linearly increases with $N$ and $m=N / 2$.

If one strand in the cable is quenched for any reason, the stability criteria may be formulated as follows: the current rise in other strands should not exceed the critical value, i.e.

$\frac{I_{\mathrm{str}}}{m} \leq I_{\mathrm{c}}-I_{\mathrm{str}}$

Because of a good inductive coupling between strands in multistrand cables the effective inductances of strands are very low. This is why all redistribution processes have a small time constant and $\mathrm{d} / \mathrm{d} t$ rates are very high ${ }^{11}$. Eventually, it leads to a reduction of the current quench level in 
Influence of cable design on stability: V. S. Vysotsky et al.
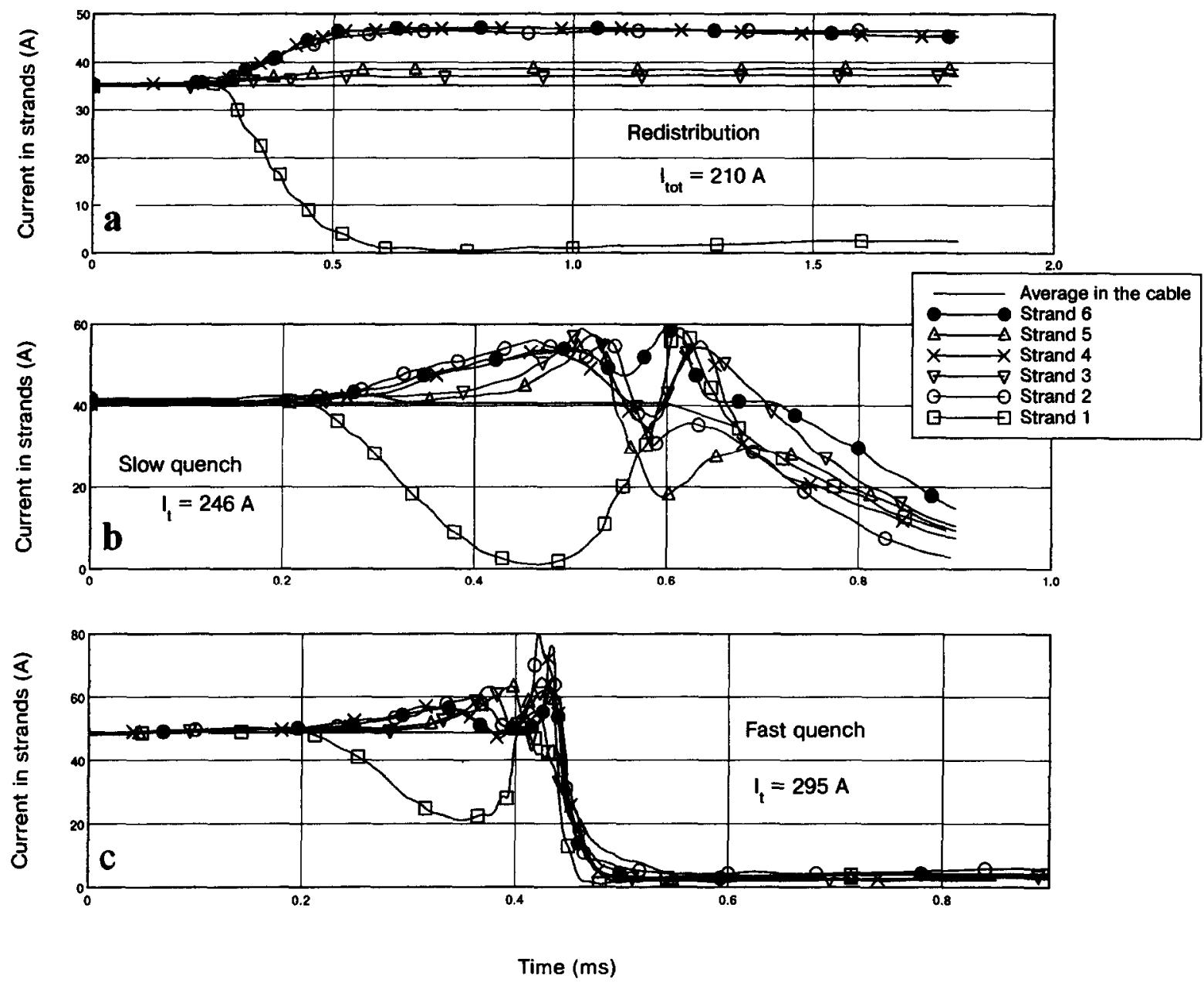

Figure 3 Quench development process in sample \#2 (round-braid cabling): (a) redistribution process: (b) slow quench process; (c) fast quench process
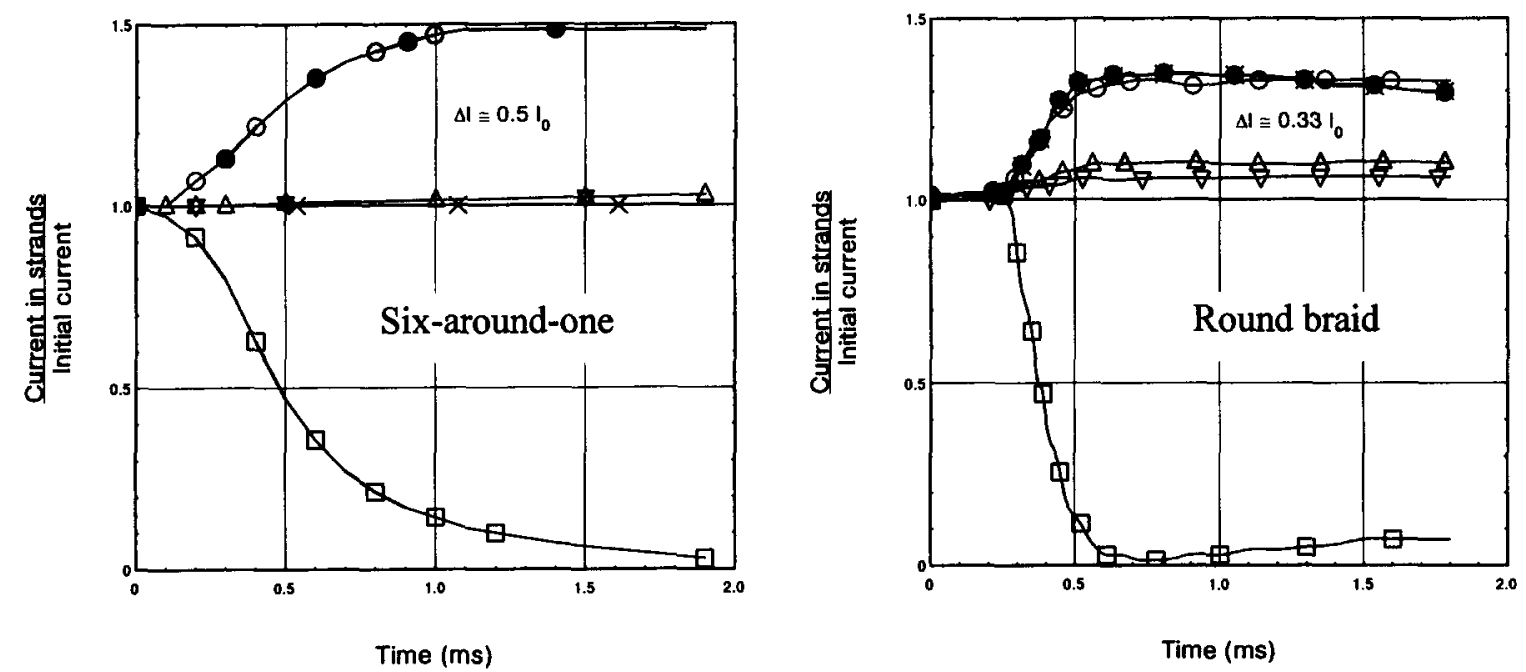

Figure 4 Redistribution of the currents in the samples, if current is normalized on the initial current value. The relative rise of currents in adjacent strands is more in the six-around-one cable than in the round-braid cable. Symbols as in Figures 2 and 3

strands with fast rising current ${ }^{4,12}$. Thus, Equation (1) should be modified to:

$$
\frac{I_{\text {str }}}{m} \leq \alpha I_{c}-I_{\text {str }}
$$

where $\alpha<1$ is a coefficicnt that detcrmines the quench current reduction due to fast current changing.
By normalizing all currents to $I_{\mathrm{c}}$ one can obtain the relative threshold current as a current level beyond which a multistrand superconducting cable will be electrodynamically unstable:

$i_{1} \leq \frac{\alpha m}{1+m}$ 

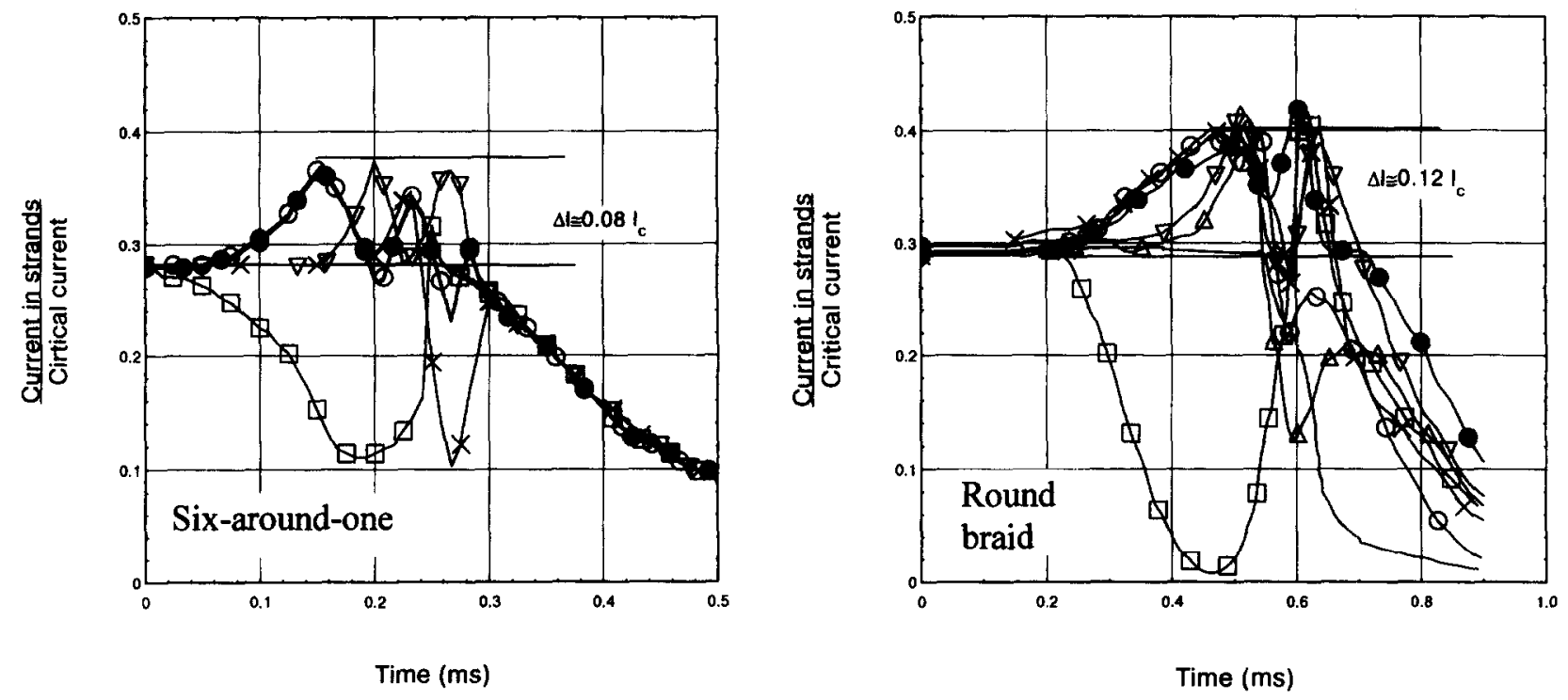

Figure 5 Slow quench development process in the samples if current is normalized on the critical current value. Possible rise of currents before quench occurs in adjacent strands is more in the round-braid cable than in the six-around-one cable. Symbols as in Figuros 2 and 3

This expression is the criteria of SS stability: the relative level of an operating current should be less than $i_{t}$. Two important conclusions about SS stability may be drawn from consideration of Equation (3).

The first conclusion is that the increase in the number of strands in a multistrand cable does not improve SS stability unless $m$ is increasing with $N$. Increasing $N$ does not improve the SS stability for usual multiple twisted cables and for Rutherford type cables. For round-braid type cables increasing $N$ may increase stability. If the number of strands is increased from six to, say, $18, i_{\mathrm{t}}$ increases by 1.2 times the original value.

There could be some cabling techniques that may provide equal mutual inductances between all strands in the cable and therefore current distribution in all but one cable's strands if this one strand quenched, e.g. as in the flat braid cable ${ }^{13}$. Development of multistrand superconducting cables with equal mutual inductances between all strands will improve their SS stability and also their general stability. This is a possible method to solving the current degradation problem in multistrand superconducting cables.

The second conclusion is that, in the best case, the relative quench current cannot exceed $\alpha$. This parameter depends on possible $\mathrm{d} I / \mathrm{d} t$ rates that may occur in the cable. Buznikov et al. ${ }^{14}$ carried out an analysis of $\alpha$ for a twostrand cable, i.e. for $m=1$. It was shown that the threshold current between redistribution and slow quench is increasing with cable length. This agrees with known experimental facts about better stability of longer multistrand superconducting cables. Possible $\mathrm{d} I / \mathrm{d} t$ levels in the cable also depend on mutual inductance between strands and it again raises the question about proper cabling to improve the SS stability. In the future we intend to analyse the influence of cable design on the parameter $\alpha$ and SS stability.

Our test samples are rather short and $\mathrm{d} I / \mathrm{d} t$ values for strand currents are $\sim 10^{4}-10^{5} \mathrm{~A} \mathrm{~s}^{-1}$. At these fast current change rates the adiabatic stability criteria should be used ${ }^{8}$ to estimate the parameter $\alpha$. In our cases it appears $\alpha$ $0.33-0.37$ that led to the observed low level of $i_{\mathrm{t}} \sim$ $0.22-0.29$.

The low stability level that was observed in coils made from multistrand superconducting cables with insulating strands ${ }^{15}$ may be explained by the high vulnerability of cables with insulated strands to a quench of a single strand or by low levels of SS stability. In cables with non-insulated strands the SS instability may be diminished by the opportunity of direct current sharing from quenched to nonquenched strands. Nevertheless, all the ideas discussed above should be taken into account during stability considerations, especially for cables with strands in highly resistive matrix or with chromium covering. Special cabling design with equal mutual inductances between all strands will improve their stability.

\section{Conclusions}

The current redistribution in two six-strand superconducting cables with different cabling type was studied for the case when one strand was deliberately quenched by the heater. A significant difference was observed in the current redistribution among strands. In the six-around-one cable, current from the quenched strand redistributed in two adjacent strands, while in the round-braid cable, current redistributed in three strands from opposite triplets. This is cxplained by the difference of mutual inductances between strands in the cables with different design.

On the basis of experimental data the new conception of single-strand stability, i.e. stability in relation to quench of one single strand of a multistrand superconducting cable, was developed. The criteria of single-strand stability was proposed. A possible way to improve multistrand superconducting cable stability is the use of a cabling method that provides equal mutual inductances between all strands.

\section{Acknowledgements}

One of the authors (V.V.) would like to thank Professor K. Funaki for useful discussions, support and encouragement to complete the work for this paper in Kyushu University.

\section{References}

1. Tsukamoto, O., AC superconductivity application for general purposes. Proc. Workshop on AC Superconductivity, 1992, pp. 44-50. 
2. MIT Plasma Fusion Center, Report PFC/RR-92-1, 1992.

3. Tompkins, J., Haddok, C. and Snitchler, G., SSC Report SSCL-SR1235, 1994, Chapter 31.

4. Vysotsky, V.S., Takayasu, M., Michael, P.C., Schultz, J.H., Mineryini, J.V. and Jeong, S., Current redistribution in a 12 strand $\mathrm{Nb}_{3} \mathrm{Sn}$ CICC and its influence on ramp rate limitation. IEEE Trans. Applied Superconductivity 1997, 7, 774-777.

5. Vysotsky, V.S., Tsikhon, V.N., Mulder, G.B.M. and Van De Klundert, L.J.M., Quench development in a superconducting cable having insulated strands with high resistive matrix (parts 1 and 2). IEEE Trans. Magn., 1992, 28, 735-742.

6. Ito, D. et al., Development of a $500 \mathrm{kVA}$ AC superconducting coil. Proc. ICEC-12, Southampton, 1988, pp. 719-723.

7. Iwakuma, M., Kanetaka, H., Tasaki, K., Funaki, K., Takeo, M. and Yamafuji, K., Abnormal quench process with very fat elongation of normal zone in multistrand superconducting cables. Cryogenics, 1990, 30, 686-692.

8. Rakhmanov, A.L., Gurevich, A.V. and Mints, R.G., The Physics of Composite Superconductors. NAUKA, Moscow, 1987 (in Russian); Begell House, New York, 1997 (in English).

9. Rusinov, A.I., Karasik, V.R. and Krivolutskaja, N.V., Analysis of electromagnetic processes in superconducting solenoids. Proc. Lebedev Physical Institute, Vol. 121, 1980, pp. 52-75 (in Russian)

10. Vysotsky, V.S., Takayasu, M., Michael, P.C., Schultz, J.H., Minervini, J.V. and Jeong, S., Measurements of current distribution in a 12 strand $\mathrm{Nb}_{3} \mathrm{Sn}$ cable-in-conduit conductor. Cryogenics, in press.

11. Vysotsky, V.S., Mulder, G.B.M., Van De Klundert, L.J.M. and Krooshop, H.J.G., Quench characteristics of a two-strand superconducting cable and the influence of its length. IEEE Trans. Magn., $1992,28,743-746$.

12. Vysotsky, V.S. and Tsikhon, V.N., The quench velocity in multifilament superconductor after fast current increase. Cryogenics, 1992, 32(ICEC Suppl.), 435-437.

13. Wilson, M.N., Superconducting Magnets. Clarendon press, Oxford, 1983, Chapter 12

14. Buznikov, N.A., Pukhov, A.A., Rakhmanov, A.L. and Vysotsky, V.S., Current redistribution between strands and quench process in a superconducting cable. Cryogenics, 1996, 36, 275-281.

15. Shimamoto, S., Okuno, A., Ando, T. and Tsuji, H., Development of superconducting pulsed poloidal coils in JAERI. Cryogenics, 1990 30(ICEC Suppl.), 23-30. 